\title{
NEEDS AND APPROACH FOR COMBINING FORMAL ANALYSIS AND HUMAN INSTINCT IN LOGISTICS SIMULATION PROJECTS
}

\author{
Gaby Neumann \\ Technical University of Applied Sciences Wildau \\ 15745 Wildau, Germany \\ E-mail: gaby.neumann@th-wildau.de
}

\begin{abstract}
KEYWORDS
simulation output interpretation, trace file analysis, simulation knowledge, logistics simulation project, knowledge-based simulation
\end{abstract}

\section{ABSTRACT}

On one hand the roles of the simulation modeller and user in logistics simulation projects seem to be pretty clear and well accepted; on the other hand general and domain-specific approaches to formalise certain steps in a simulation project return to be the subject of research and development. Typically those approaches head for software engineering and tool development in order to replace the person in model building steps or most recently in trace file analysis. Unfortunately, this quite often relinquishes and intuition of the simulating person. Against this background the paper draws attention to the benefits from combining both spheres, the one of formalized algorithms and the other one of the human instinct. Based upon a discussion of simulation user needs in the logistics application area an approach is presented that allows mediating between user and software in both ways, for specifying simulation aims and questions and for deriving simulation results from seeing behind pure simulation data. By use of an example the impact the user has on a simulation project and its outcomes is illustrated. Consequently, conclusions emphasize the irreplaceable role of the user who brings in objectives, motivation and focus of the simulation project as well as domain-specific experiences and competences to understand the real message of simulation results.
\end{abstract}

\section{INTRODUCTION AND MOTIVATION}

One of today's challenges consists in seeing simulation in the context of human-centred processes. In recent literature this is being addressed by, for example, providing simulation modellers (or users) with methods and tools for automatic trace file analysis in order to better cope with large amounts of simulation output data. Those approaches mainly focus on formalizing simulation outcome in the context of a certain application area.

Kemper and Tepper (2009), for example, state that in tracing a simulation model a modeller finds himself in the situation where it is unclear what properties to ask to be checked by a model checker or what hypothesis to test. They assume that cyclic behaviour of model components is always good behaviour whereas all exceptions or disturbances in this behaviour indicate errors. Therefore, the aim is to provide support by automatically identifying and removing repetition from a simulation trace in order to pay particular attention on the non-returning, progressing part of a trace. This is to be achieved by automatic trace file reduction as it is assumed that modellers do not have enough background knowledge or experience to figure out interesting parts of the trace themselves.

Wustmann, Vasyutynskyy and Schmidt (2009) assume that simulation usually aims to specify whether or not the concept of a material flow system meets formal requirements, but not how well it does it. This is said to be caused in limited methodological support and therefore strongly depend on the modelling/planning expert's experience and expertise. This is to be overcome by eliminating the user as weakest point through automatic analysis. For this an analysis tool is proposed that helps in identifying the concept's or the system's weak points, specifying their primary reasons and pointing out system immanent potential for performance increase.

Both approaches have in common the very much reduced role they give to the key actor(s) in any simulation project: the person who builds the simulation model and the person who uses the simulation model to run experiments. Instead they assume any result derived from simulation can directly and automatically be extracted from the trace file through statistical analysis, clustering or reasoning without any additional explanation by the simulating person. If this would be the case then any simulation model and any plan of experiments can be seen as objective representation of a particular part of reality and its problem situation. Any model building or experimentation activity no matter what background or intention one has would lead to the same model and to the same collection of simulation output. A particular simulation output always would lead to the same conclusions, i.e. simulation results, no matter what is being analyzed by whom and how.

If this would be the case, why do simulation projects still require involvement of human resources of certain 
expertise? It is because simulation projects are not only sequences of formalizing steps that can be fully represented by more or less complex logical algorithms, but also require intuitive problem solving, combining analyzing steps and the need for creative thinking. Whereas the first can already be formalized or will be in future, the latter always remains linked to the person carrying out or contributing to or requesting simulation projects. Approaches to increase the degree of formalization in simulation, no matter if they focus on automatic model generation or automatic trace file analysis and simulation result delivery, will always be limited by the impossibility of fully formalizing the objectives and goals of a simulation. As already concluded by Helms and Strothotte (1992) the simulation user will therefore continue to be the key factor in any simulation project.

Against this background the following sections of the paper discuss the role the user plays in simulation projects and gives proof of the impact the user has on the simulation results achieved different types of users with individual background, experience and intention build individual simulation models and run simulation experiments for the same problem. Comparative analysis investigates differences and similarities of models, experiments, results and findings achieved by the different users. Further on, it is specified what domain experts expect to find from simulation studies using logistics as exemplary application area and a methodology is proposed for supporting those users in specifying and achieving output data needed. The paper ends with summarizing research findings and presenting conclusions derived from them.

\section{HUMAN IMPACT IN SIMULATION PROJECTS}

In general, simulation projects in the field of logistics as in other fields too - are organized in the form of a service involving both, simulation experts and logistics experts with individual knowledge to be of use at certain stages of the project: Simulation experts are primarily responsible for model building and implementation steps, whereas logistics experts mainly provide application-specific knowledge for problem description, identification of input data and evaluation of results (Neumann and Ziems 2002). In order to better understand the role of the user in simulation it is worth to take a closer look at simulation knowledge sources and stakeholders for identifying which knowledge comes from where and in which form.

In general, input information for a simulation project usually come with the tender specification or are to be identified and generated in the problem definition and data collection phases of the simulation (Figure 1). Here, the user decide (and bring in) what is to be taken into consideration for model building and which information is required for the investigation.

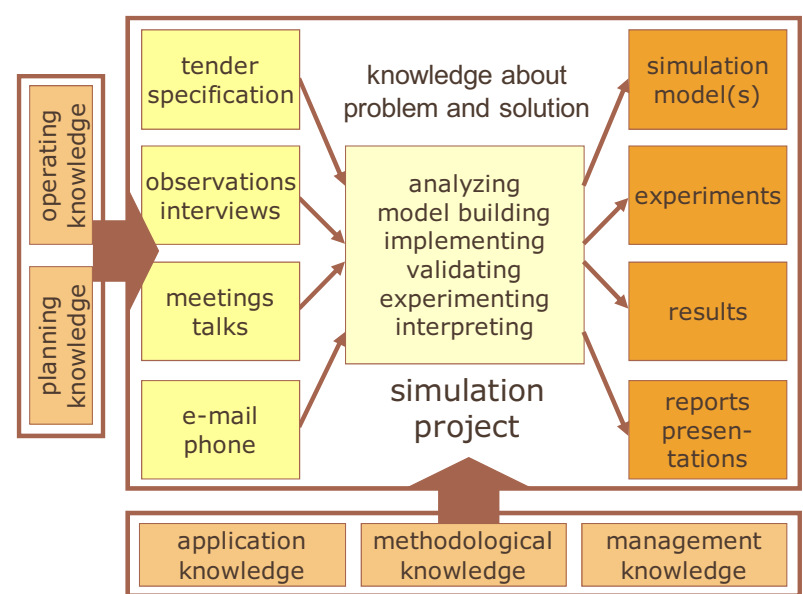

Figure 1: Sources and evolution in simulation knowledge

The model-building process should be seen as another important phase of collecting, evaluating and structuring information. As discussed by Neumann (2007) a simulation model is more than just a tool necessary to achieve certain objectives of experimentation and cognition. In the course of a simulation project the simulation model is developed, modified, used, evaluated and extended within an ongoing process. Therefore, it is also a kind of dynamic repository containing knowledge about parameters, causal relations and decision rules gathered through purposeful experiments. Even further, knowledge is "created" systematically through simulation based on the systematic design of experiments (including a meaningful definition of parameters and strategies) and the intelligent interpretation of results.

Simulation experiments, for example, to support logistics planning and operation might be oriented towards modifications in either functionality or structure or parameters of a model and its components or even in a combination of those variations leading to more complex fields of experiments. Experimentation efforts are directly related to the type of variation required. The latter depends on the specific design of the simulation model resulting from the underlying modelling concept of the simulation tools and the design of the conceptual model by its developer. To correctly interpret simulation output it is necessary to understand what the objectives, parameters and procedures of a certain series of experiments were and to relate this to the results and findings. Consequently, the objectives of a simulation and the questions to be answered by experiments should already be taken into consideration when designing the conceptual model. Specific opportunities and features offered by the selected simulation tool then influence transformation of the conceptual model into the computer model when it comes to model implementation.

All steps again and again require input and background information based upon the knowledge and experience of the users, i.e. the simulation expert and the domain 
expert. In terms of simulation target definition it is particularly necessary to understand what the domain expert expects from simulation. As this is typically specific to the application area, we continue discussions using logistics as example.

\section{CASE STUDY ON THE IMPACT OF THE USER}

In order to demonstrate the role of the user in a simulation project and his/her impact on simulation results at both levels, the one dealing with (abstract) data and the one related to the user's point of view, the same problem description and input data can be given to different types of users for building the simulation model, running experiments and deriving simulation results. These users might vary in their domain-specific background (e.g. logistics or computing expert, engineer or management person, simulation service provider) and experience (e.g. novice or expert), but they all run the full simulation project including typical phases like problem analysis, model building, implementation, validation, experimenting and interpretation. For this, it is left up to them which analysis or modelling tools or simulation package they might use. Based upon the finalized project comparative analysis can run in order to understand what findings and recommendation from the simulation have in common and what differences are caused by the individual approaches. With this, we can demonstrate what might happen if user-specific motivation and intention for simulation modelling and experiments in a particular case is not taken into consideration when deriving conclusions from statistically analyzing trace files only.
A first example of such a comparative case has been run and analysed by Neumann and Page (2006). Here, the same logistics simulation problem, namely to identify the performance limit of two different designs and two varying operational scenarios for automated stacking at high-performance container terminals has been given to two individually operating groups of students from different domains (Figure 2). The first group was composed of computing students from the University of Hamburg, whereas the second group was formed by logistics students from the University of Magdeburg. All students had already a certain simulation background corresponding to their educational profile.

The main differences between the approaches in both cases consist in the preparation of model implementation and the simulation models themselves. The logistics students started with a detailed analytical investigation of the system and process to gain a clear understanding of the situation and more detailed specification of the problem. From this they derived a conceptual model which they had in their minds when starting to implement the model using the DOSIMIS-3 simulation package, but they did not document it in any formalized way. Due to the fact that they used predefined building blocks for model implementation already representing a particular amount of functionality and logic, they had to deal with certain limitations (or at least special challenges) in modelling and therefore in advance spent many thoughts on what really is needed to be represented in the simulation model at what level of detail. This led to a number of simplifications such as the representation of the stacking cranes' movements on the basis of a detailed understanding of the material flow backgrounds of the simulation problem.

\begin{tabular}{|l|c|c|c|}
\hline & $\begin{array}{c}\text { Saanen and } \\
\text { Valkengoed } \\
(2005)\end{array}$ & $\begin{array}{c}\text { Computing } \\
\text { students }\end{array}$ & $\begin{array}{c}\text { Logistics } \\
\text { students }\end{array}$ \\
\hline Twin & $49 / 53^{*}$ & $45(\mathrm{~d})$ & $55.3(\mathrm{~d})$ \\
RMGs & & $47.9(\mathrm{n})$ & $47.6(\mathrm{n})$ \\
\hline Cross- & $49 / 53 / 45^{*}$ & $49.4(\mathrm{~d})$ & $50.6(\mathrm{~d})$ \\
over & & $68(\mathrm{n})$ & $61.5(\mathrm{n})$ \\
RMGs & & & \\
\hline
\end{tabular}

d/n = day-time/night-time scenario

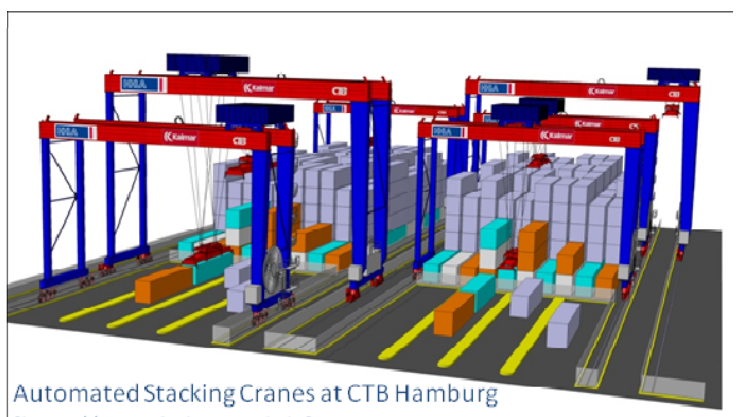

[http://www.kalmarind.de]
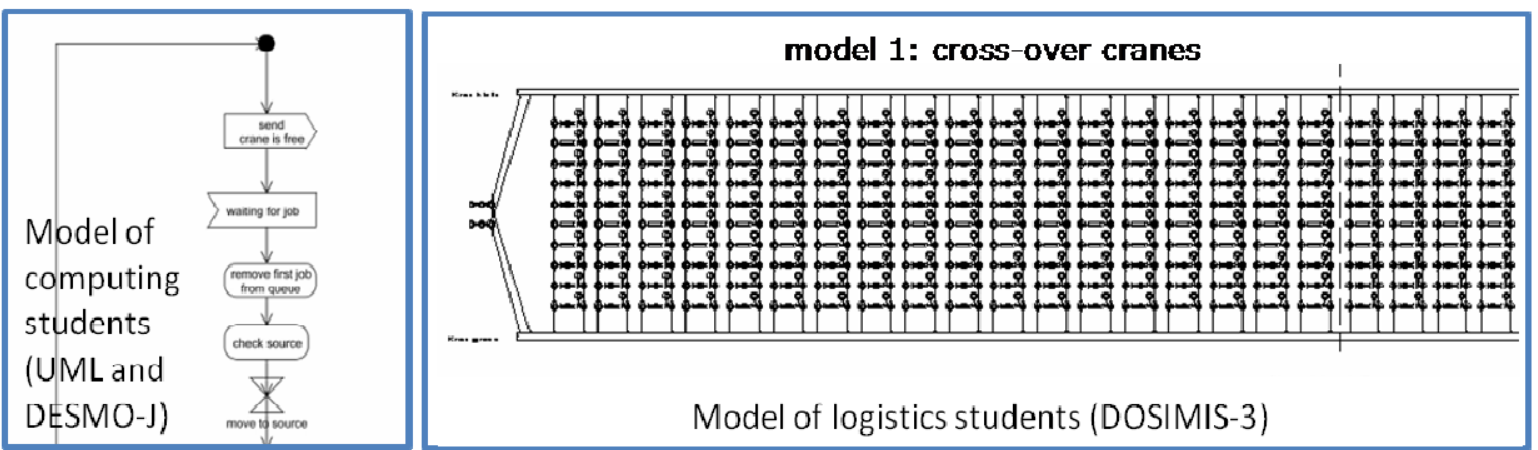

Figure 2: The impact of the user: a case study 
In contrast to this the computing students focused on a very detailed representation of the stacking cranes' movements including precise tracking of the cranes' positions while moving, but they set aside representation of the storage places and individual containers. This also required detailed modelling of the cranes' management and control system and algorithms, whereas neither warehouse nor stock management was needed. Although these students had not to deal with the large number of storage locations, detailed representation of the stacking cranes required a lot of extra efforts, especially because of the complex and complicated control algorithms. Here, no limitations nor specific restrictions have been set by the simulation tool used (Java-based programming within the DESMO-J framework) and therefore the students were not forced to re-think about what really would be required to be represented in the model.

In the end both student projects produced valid and usable simulation models, but efforts for model implementation, in the course of experimentation eventually needed modification and visualization of results were quite different. Although the students were not highly experienced simulation experts which of course had an influence on the effectiveness of the model building process, those different modes of approaching and solving a simulation problem can be found in more professional simulations, too.

Results achieved from either model allowed responding to the initial question for the performance limit in terminal operation. Comparison of those results showed just some slight deviations which possibly were caused by differences in some basic technical and layout parameters, such as crane speeds and stacking module dimensions, due to different assumptions. In addition to this varying storage/retrieval strategies have been used. Although the total of these differences is consequently represented by the deviation of results, it also can be stated that results are quite similar. This finally allows concluding that despite of different modelling approaches similar simulation results could be achieved.

Nevertheless, different modelling approaches and simulation tools used resulted in very different ways of achieving the intended outcome. Simulation models were quite as individual as resulting trace files were especially what concerns level-of-detail. Therefore, any standardized or formalized approach for trace file analysis and interpretation of output without involving the model developer (and at the same time user in this particular case) would have failed or delivered very general (rough) results only.

This first case study in an educational setting can just be the starting point for more detailed investigations on the impact a user's individual background and even personality might have on the design, implementation and use of a simulation model. In order to get a better insight and eventually derive some stereotypes further case studies need to be run that involve professional simulation users of different backgrounds.

\section{EXPECTED OUTCOMES FROM LOGISTICS SIMULATION PROJECTS}

In the course of a logistics simulation project both partners, logistics expert (simulation customer) and simulation expert (simulation service provider), use to face the ever challenging task to interpret numerous and diverse data in a way being correct with respect to the underlying subject of the simulation study and directly meeting its context. These data are usually produced and more or less clearly presented by the simulation tool in the form of trace files, condensed statistics and performance measures derived from them, graphical representations or animation. Problems mainly consist in:

1. clearly specifying questions the simulation customer needs to get answered,

2. purposefully choosing measures and selecting data enabling the simulation service provider to reply to the customer's questions, or

3. processing and interpreting data and measures according to the application area and simulation problem.

To overcome these problems and give support in defining simulation goals and understanding simulation results, methods and tools are required that are easy to use and able to mediate between knowledge and understanding of the simulation customer (the logistics expert planning or operating that process and system to be simulated) and the simulation expert (the expert from the point of view of data and their representation inside computers). Within this context, it is worth thinking in more detail about what a simulation customer (the logistics expert) might look for when analyzing the outcome of simulation experiments (Neumann 2005):

- Typical events. The logistics expert specifically looks for moments at which a defined situation occurs. This kind of query can be related, for example, to the point in time at which the first or last or a specific object enters or leaves the system as a whole or an element in particular. Other enquiry might be oriented towards identifying the moment when a particular state or combination of states is reached or conditions change as defined.

- Typical phases. The logistics expert is especially interested in periods characterized by a particular situation. In this case $\mathrm{s} / \mathrm{he}$ asks for the duration of the warm-up period, for the period of time the system, an element or object is in a particular state, or how long a change of state takes. 
- Statements. The logistics expert looks for the global characteristics of processes, system dynamics or object flows such as process type (e.g. steady-state, seasonal changes, terminating/non-terminating), performance parameters of resources (e.g. throughput, utilization, availability), parameters of object flows (e.g. mix of sorts, inter-arrival times, processing times). This information is usually based on statistics resulting from trace file analysis and replies to either a specific or more general enquiry by the user.

\section{APPROACH FOR MEDIATING BETWEEN USER AND MODEL IN A SIMULATION PROJECT}

When the potential interests of a simulation customer as explained above are compared, one significant difference emerges: whereas the first two aspects need specific questions formulated by the logistics expert directly at data level, the last aspect is characterized by usually fuzzy questions of principle from the more global user's point of view. Before these questions of principle can be answered, they have to be transferred to the data level by explaining them in detail and putting them in terms of concrete data (Figure 3).

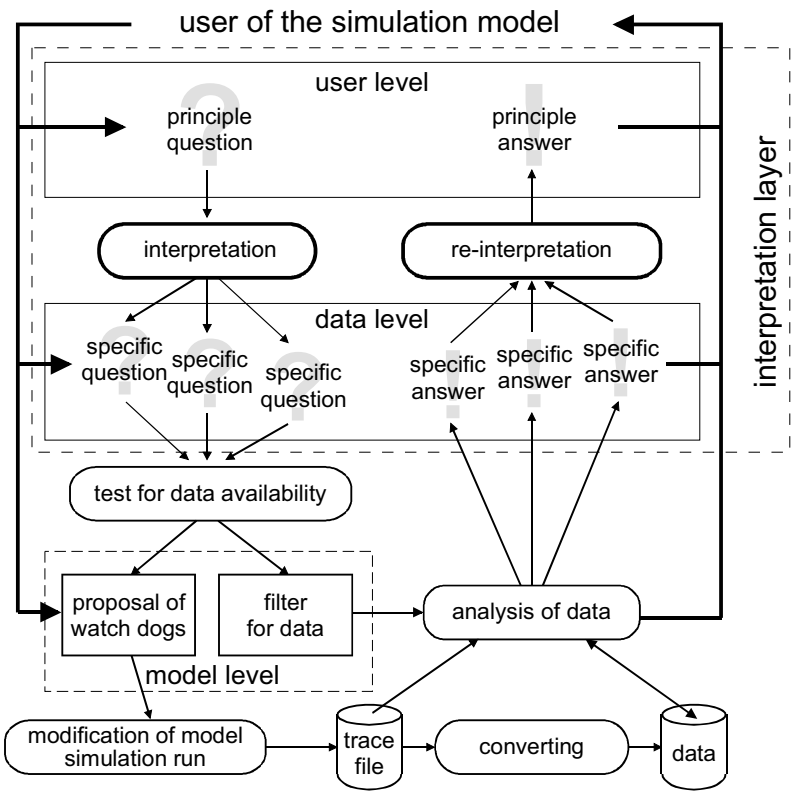

Figure 3: User-data interaction for simulation output analysis

As result of this process of interpretation a set of specific questions is defined with each of them providing a specific part of the overall answer in which the user is interested. Questions at data level correspond to results that can be delivered directly by the simulation even if minor modifications to the simulation model should be required (Tolujew 1997). This is the kind of study also current approaches for trace file analysis support (Kemper and Tepper 2009; Wustmann, Vasyutynskyy and Schmidt 2009). To derive an answer in principle to a question of principle the respective set of specific answers needs to be processed further. These steps of additional analysis and condensing can be understood as a process of re-interpretation to transfer results from data to user level.

All steps of interpretation and re-interpretation aim to link the user's (logistics expert's) point of view to that of the simulation expert. They not only require an appropriate procedure, but, even more importantly, an interpretative model representing the application area in which simulation takes place. This model needs to be based on knowledge and rules expressed in the user's individual expertise, but also in generalized knowledge of the (logistics) organization regarding design constraints or system behaviour and the experience of the simulation expert derived from prior simulations. As this knowledge might not only be of explicit nature, i.e. existing independent of a person and suitable to be articulated, codified, stored, and accessed by other persons, but also comprise implicit or tacit knowledge carried by a person in his or her mind often unawares, simulation users as individuals or team need to remain involved in the steps of interpretation and reinterpretation at least. Whereas explicit knowledge might be transferred into rules and algorithms, tacit knowledge cannot be separated from its owner and therefore requires direct involvement of the knowledge holder in the interpretation process. More specifically this means support is required for translating any principle question into corresponding specific (datarelated) questions as well as for deriving principle answers from a number of specific (data-related) answers. Although a set of (standard) translation rules might be known, formalized and put into the rule base already, always further questions remain that are unknown to the rule base yet. Here, the logistics expert needs support in

1. correctly formulating the right question and

2. getting the full picture from the puzzle of available data and their analysis.

One approach for enabling this could be based on viewpoint descriptions. Viewpoint descriptions were introduced into model validation as a new kind of communication and interaction between the human observer of simulation results and the computer as the simulation model using authority that was called oraclebased model modification (Helms and Strothotte 1992). Here, the principle idea is that the user presents his or her observations (in the animation) as a viewpoint description to the computer that initiates a reasoning process. This results in definition and realization of necessary changes to the simulation model in an ongoing user-computer dialogue. The main advantage of this concept lies in the reduced requirements for rulebase definition. Those aspects that easily can be formalized (e.g. typical quantitative observations or unambiguous logical dependencies) are translated into 
questions to the user (What is it $\mathrm{s} /$ he is interested in?) or various forms of result presentation (as figures or diagrams), whereas those that are non-imaginable yet or individual to the user or simply hard to formalize need not to be included to provide meaningful support to the user. There is no need to completely specify all possible situations, views and problems in advance, because the person who deals with simulation output brings in additional knowledge, experience and creativity for coping with non-standard challenges. Even further, this way the rule-base continuously grows as it "learns" from all applications and especially from those that were not involved yet. On the other side the user benefits from prior experience and knowledge represented in the computer by receiving hints on what to look at based upon questions other users had asked or which were of interest in earlier investigations.

This approach helps in designing the interpretation layer for mediating between simulation customer and simulation model or output no matter how many data have been gathered and how big the trace file grew. Nevertheless, effectiveness and efficiency of this interpretation process depends on the availability of the right data at the right level of detail. This quite often does not only depend on the simulation model and tool used for its implementation, but also on the opportunity to aggregate data in always new ways.

As discussed simulation results derived from running experiments by use of a particular simulation model are as good as they finally respond to the questions the simulation user is interested in. The challenge consists in knowing about questions a user in a specific project might have. Generally, a certain amount of (standard) questions can be pre-defined in correspondence with the application area and another set of questions might be defined by the user when starting into simulation modelling and experimentation. This might even lead to a specific focus in trace file generation and recording of simulation output data by purposefully introducing a cohort of observers to the model that directly correspond to the type and amount of data required for responding to questions already addressed by the user (Tolujew 1997).

However, it is not that exceptional that new questions arise in the cause of the simulation project when seeing results from previous experiments. In those situations it might either be necessary to re-run simulation with a modified observation concept or to aggregate or derive results from already existing simulation output in a different way. Concerning the first, there are two options for interpreting simulation output: online and offline (Tolujew et al. 2007).

\section{Online interpretation might focus on:}

- visualizing changes in the position of moving objects;

- $\quad$ visualizing states (e.g. stock development);
- identifying or recognizing pre-defined situations.

Offline interpretation typically is used for:

- calculating freely definable characteristics;

- identifying or recognizing pre-defined situations;

- preparing and showing special-focused animations.

Although being specific to a certain simulation project, those analysis steps are possible to be pre-specified and also in the focus of approaches as presented by Kemper and Tepper (2009) and Wustmann et al. (2009). But beyond this, specific questions relevant in a certain simulation project might eventually even require to summarize (primary) objects as simulated into new (secondary) classes not simulated yet. In a transportation model with a number of trucks moving different types and different volumes of goods, for example, it suddenly might be of interest to know something about all those trucks arriving Tuesdays only. The simulation model itself knows trucks as one class of objects, but does not contain "Tuesday trucks" as a specific sub-class to this. This new class needs to be formed out of the situation and might then be added to the rule-base for trace file analysis, but cannot be predefined as simply not specified before. Consequently, any tool to support trace file analysis must allow and even support those interactions with the trace file which again goes far beyond formal statistical analysis.

\section{CONCLUSIONS}

To understand the message of simulation results formal trace file analysis is one important step. The other one is the non-formal, more creative step of directly answering all questions that are of interest to the user (in our case the logistics expert). The precondition is to know (and understand) what the questions of the user are, but also the ability of the user to ask questions relevant to a particular problem. For the latter, the framework for trace file analysis and interpretation provides even further support: Typical questions no matter if they are of generic or specific nature help the user in identifying the problem or the questions to be asked or the aspects to be investigated. As discussed, this can be supported by the approaches for viewpoint description and defining observers or specifying analysis focus. Additionally, a pattern combining typical symptoms (i.e. visible situations or measurable characteristics) with the underlying problems causing those symptoms would be of huge benefit as this might also guide the user in truly understanding what happens in a specific material handling or logistics system.

Current approaches to trace file analysis mainly focus on deriving (standard) parameters and (typical) characteristics by use of statistical methods, clustering or reasoning. With this they provide results at data level 
(Figure 3) allowing basic interpretation based upon (externalized) domain-specific knowledge. This step works automatically for those aspects that can be formalized and shows limited results only for those aspects that require intuitive thinking by the user.

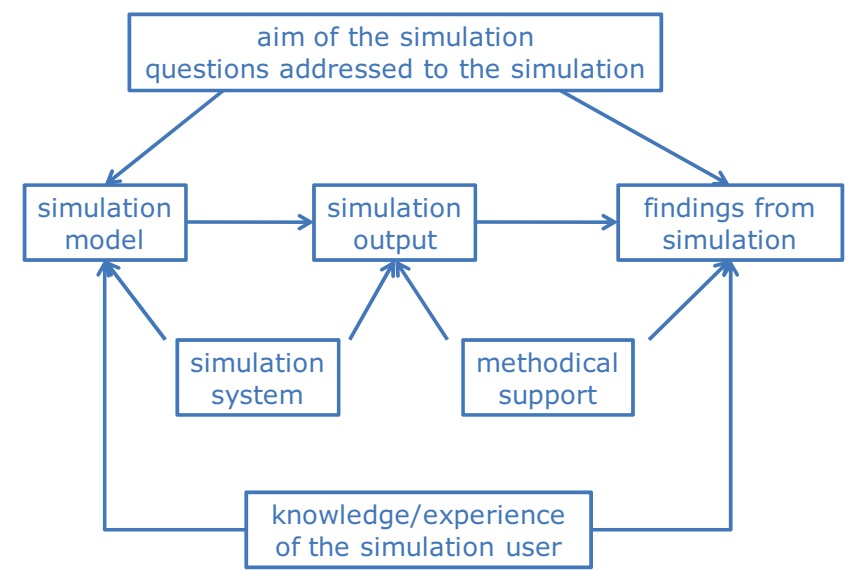

Figure 4: Impact of the simulation user on the outcome of a simulation project

Against this background the paper concludes that it is necessary to see behind the simulation results by interpreting simulation output in order to understand their real message. This interpretation requires knowledge and understanding of the domain/ application area as well as mathematical and statistics skills. Trace file analysis supports preparation of interpretation steps but cannot fully replace the user who brings in objectives, motivation and focus of the simulation project as well as domain-specific experiences and competences to understand the message of simulation results (Figure 4). A sophisticated framework especially helps to reduce routine work like statistics calculations through incorporated powerful analysis tools and stimulating creative thinking by proposing, asking, suggesting in a really interactive communication between the simulation user and the computer.

\section{REFERENCES}

Helms, C., Strothotte, T., 1992. Oracles and Viewpoint Descriptions for Object Flow Investigation. Proc. of the 1st EUROSIM Congress on Modelling and Simulation, 47-53, Capri, Italy.

Kemper, P., Tepper, C., 2009. Automated trace analysis of discrete-event system models. IEEE Transactions on Software Engineering, vol. 35, no. 2, 195-208.

Neumann, G., 2005. Simulation and Logistics. In: Page, B., Kreutzer, W., eds. The Java Simulation Handbook Simulating Discrete Event Systems in UML and Java. Aachen: Shaker, 435-468.

Neumann, G., 2007. The role of knowledge throughout the simulation lifecycle: what does a simulation model know? Proc. of the 6th EUROSIM Congress on Modelling and Simulation, vol. 2, TH-1-P12-5, Ljubljana, Slovenia.
Neumann, G., Page, B., 2006. Case study to compare modelling and simulation approaches of different domain experts. Proc. of I3M 2006 - International Mediterranean Modelling Multiconference, 517-522, Barcelona, Spain.

Neumann, G., Ziems, D., 2002. Logistics Simulation: Methodology for Problem Solving and Knowledge Acquisition. Proc. of the 6th Multiconference on Systemics, Cybernetics and Informatics, vol. XII, 357-362, Orlando/Florida, USA.

Tolujew, J., 1997. Werkzeuge des Simulationsexperten von morgen. Proc. of Simulation and Animation '97, 201-210, Magdeburg, Germany (Tools of the simulation expert of tomorrow, in German).

Tolujew, J., Reggelin, T., Sermpetzoglou, C., 2007. Simulation und Interpretation von Datenströmen in logistischen Echtzeitsystemen. In: C. Engelhardt-Nowitzki; O. Nowitzki; B. Krenn (editors): Management komplexer Materialflüsse mittels Simulation. State-of-the-Art und innovative Konzepte. Leobener Logistik Cases, Wiesbaden: Deutscher Universitäts-Verlag, S. 215-232. (Simulation and interpretation of data streams in logistics real-time systems, in German)

Wustmann, D., Vasyutynskyy, V., Schmidt, T., 2009. Ansätze zur automatischen Analyse und Diagnose von komplexen Materialflusssystemen. Proc. of the 5th Expert colloquium of WGTL - Wissenschaftliche Gesellschaft für Technische Logistik, 1-19, Ilmenau, Germany (Approaches for automatic analysis and diagnosis of complex material flow systems, in German).

\section{AUTHOR BIOGRAPHY}

GABY NEUMANN received a Diploma in Materials Handling Technology from the Otto-von-GuerickeUniversity of Technology in Magdeburg and a $\mathrm{PhD}$ in Logistics from the University of Magdeburg for her dissertation on "Knowledge-Based Support for the Planner of Crane-Operated Materials Flow Solutions". Between December 2002 and June 2009 she was Junior Professor in Logistics Knowledge Management at the Faculty of Mechanical Engineering there. In December 2009 she became full Professor on Engineering Logistics at the Technical University of Applied Sciences Wildau. Since 1991 she also has been working as part-time consultant in material handling simulation, logistics planning and specification of professional competences in certain fields of logistics. Her current activities and research interests are linked amongst others to fields like problem solving and knowledge management in logistics, logistics simulation and planning. She organises or co-organises workshops and conferences, supports international programme committees of various conferences, and has been or is being involved in a couple of research projects in these fields. Gaby Neumann has widely published and regularly presents related research papers at national and international conferences. 